\title{
Testability of Software Components
}

\begin{abstract}
The knowledge as to whether a software component is testable or not is important to the software engineering process: a program that is not easily testable may have to go through several iterations of program and test redesign. In this paper, we formally investigate the meaning of software testability. We define a new concept, domain testability, by applying the concepts of observability and controllability to software. Observability refers to the ease of determining if specified inputs affect the outputs; controllability refers to the ease of producing a specified output from a specified input. Observability and controllability properties are already used for assessing the testability of hardware components. A domain testable program is observable and controllable: it does not exhibit any test input-output inconsistencies. We discuss the domain testability properties of several programs that have been presented in the literature, and investigate the observability and controllability properties of several programming structures. We also define new testing metrics (that can be applied to programs or to functional specifications) that can be used to easily assess the level of effort required to modify a program so that it becomes domain testable. We also show how testability can be assessed from program specifications, and discuss an experiment that shows that it takes less time to build and test a program developed from a domain testable specification than a similar program developed from a non-domain testable specification. In the Appendix, domain testability is defined in terms of denotational semantics.
\end{abstract}

\section{AUTHOR}

Roy S. Freedman

Formerly with

Department of Computer Science

Polytechnic University

333 Jay Street

Brooklyn, New York 11201

Currently with

Inductive Solutions, Inc.

380 Rector Place

New York, New York 10280

To Appear in the IEEE Transactions on Software Engineering, Vol. 17, No. 6, June, 1991, pp. 553-563. 


\section{Testability of Software Components}

\section{Introduction}

Testing is a crucial software development activity that is used for determining whether a program has errors. Testing is used to assess the compliance of a program to its intended specifications and to assess the reliability of the program to inputs that were not intended to be part of the specifications. Good test inputs are those that result in a high probability of discovering errors upon program execution.

Before testing begins it is necessary to identify the input and output data characteristics (ie, type, format, range) of the component, and the "states" of the component. During testing, the inputoutput data characteristics may be inferred from specific identifiers in the program; states and state transitions usually are not associated with specific identifiers.

Test plans are documented in a Test Design Specification [32]. This document specifies the test inputs, the expected outputs, and information related to timing, display, or the test execution environment. The actual outputs, and information such as timing or display anomalies, (and descriptions of attempts to repeat the test) are documented in the Test Incident Report [32].

Research in testing has focused on the following two problems:

$o$ Test Effectiveness: What is the best selection of test data?

$o$ Test Adequacy: How do we know that sufficient testing was performed?

Test effectiveness is concerned with generating the smallest set of input test data whose output would result in the discovery of the largest set of errors. The definition of an "effective" test was addressed by $[11,12]$ : if we can partition the input space into a finite set of input equivalence classes then an effective test is a test that executes one input from each class. The problem is in finding the partition. In the extreme case where the partition is discrete and the equivalence class consists of singletons, the effective test becomes an exhaustive test: all inputs must be executed. Exhaustive tests should be avoided on pragmatic grounds: they are combinatorially explosive, and, for programs with infinite input domains, can never be completed.

A testing strategy can be considered to be a method of generating a finite set of input equivalence classes. These methods range from functional (specification or "black-box") methods that select test inputs based on specification-derived values [20] to structural ("white-box") methods that select test inputs based on a percentage of the statements (or other structures) that can be executed [16,29]. Test adequacy is concerned with determining the effectiveness of test selection strategies. Several strategies were experimentally compared by Basili in [2]. And, given a test selection method, Weyuker [30] and Zweben[31] discuss sets of rules to determine whether or not sufficient testing has been performed.

One assumption in determining test effectiveness or test adequacy is that testing can be improved by improving the test selection strategy or the test adequacy criteria. It is assumed that all programs are "easily testable," or at least that the amount of work needed to identify the input and 


\section{Testability of Software Components}

output data characteristics and states of the component is small. The problem with this assumption is that the emphasis is on selecting input data, and not on determining whether the given program is easily testable or not. In this paper, we address the following problem:

o Testability: What are the properties of "easily testable" programs?

The knowledge as to whether a component is easily testable is important to the test planning and budgeting process: a program that is not easily testable may have to go through several iterations of test redesign.

Software engineering design methodologies have informally stated some properties of "easily testable" software components [3, 5, 6, 7, 14, 25, 27]. Some properties of "easily testable" hardware components have been also identified in [8,23]. Intuitively, a software component that is "testable" has the following desirable properties:

Test sets are small and easily generated. Exhaustive tests are infeasible. Testing pragmatics dictate finite test budgets.

Test sets are non-redundant. Repeated values for test inputs are inefficient. On the other hand, something may be seriously wrong if the same test value yields different results.

Test outputs are easily interpreted. Test cases are represented in a Test Specification [32], where inputs and the anticipated outputs are tabulated. The precise identification of these inputs and outputs is necessary for an effective and adequate test.

Software faults are easily locatable. Software faults are easily traced to specific components and inputs.

The knowledge as to whether a program is easily testable software or not is not explicitly provided to testers a priori. During testing, testers may determine that certain software components are not "easily testable" because they may exhibit one of the following Input-Output Inconsistencies:

Input Inconsistencies. If a test input value is repeated, its output result should be the same. If it is not then the test inputs are incomplete: the outputs are not functionally related to the inputs alone, but on some other states that have not been identified by the testers. For example, given software component A with the following specification-based (black-box) context:

type CODE is $(0,1,2)$ :

type COLOR is (RED, YELLOW, GREEN): function $\mathrm{A}(\mathrm{X}$ :in $\mathrm{CODE})$ return $\mathrm{COLOR}$;

An excerpt from a Test Incident Report in Table 1 shows that function A returns different values for some identical inputs. It may be that function A returns these values due to certain side- 


\section{Testability of Software Components}

effects in the execution environment. There may be undiscovered state and state transition that are not associated by the tester with specific inputs. Input inconsistency illustrates the fact that just because easily testable programs should have non-redundant test sets, it does not imply that any program has a non-redundant test set. This type of inconsistency occurs in applications involving database management systems and distributed systems.

\begin{tabular}{|cccccc|} 
& Test 1 & Test 2 & Test 3 & Test 4 & Test 5 \\
\hline Input $X:$ & 0 & 1 & 2 & 3 & 0 \\
Output $A(X):$ & RED & GREEN & RED & GREEN & GREEN \\
\hline
\end{tabular}

Table 1. Input-Output Table showing Test Anomalies

Output Inconsistencies. If an output identifier is specified to range over a domain of values, we should be able to construct a test input whose execution can "cover" any one of the specified values for the output identifier. If the component is a predicate in a conditional statement or a loop, an output inconsistency can imply the existence of unreachable statements or unreachable control paths.

Domain specifications are stated in identifier declarations, type definitions, comments, and in functional specifications. For example, the excerpt from the Test Incident Report in Table 1 shows that $\mathrm{A}(\mathrm{X})$ never returns the value YELLOW for all test inputs specified. It may be that either

- A was never specified in the functional requirements to assume YELLOW.

- A was incorrectly or incompletely specified.

- A returns the value YELLOW due to unidentified states in the execution

- A contains an infinite loop, and the tester (via the run-time environment) interrupts computation so that an interrupting value is returned. 


\section{Testability of Software Components}

An example of this last possibility seen in the call to A as in the following testing environment:

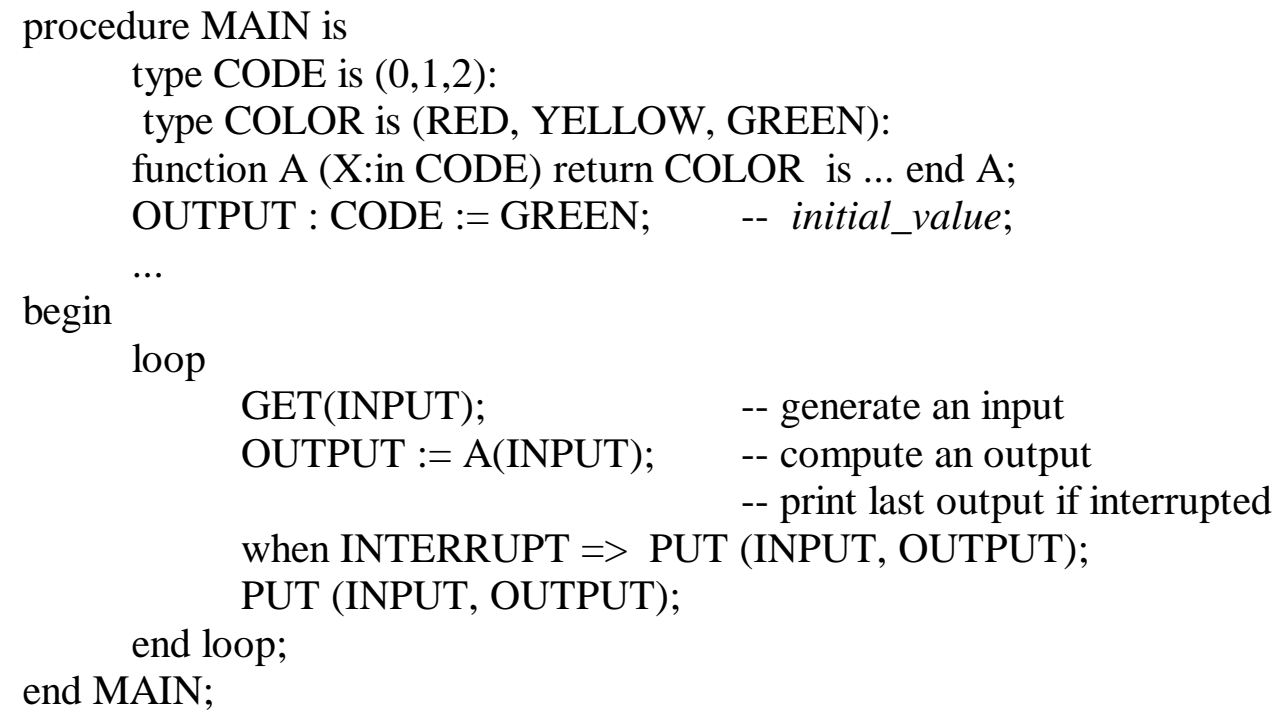

Input-Output inconsistencies lead to large, redundant test sets, where test outputs are not easily understood because of a dependency on unidentified states. Programs with input-output inconsistencies are not easily testable. In some sense, input-output inconsistencies are a consequence of using von Neumann programming languages [1]. Input-Output inconsistencies can provide evidence for program errors $[3,14,25]$, but do not imply that the program has faults: a program containing unreachable statements or an infinite loop may not be easily testable but may be correct.

In this paper, we formally define a property called domain testability as an extensional property of programs. Programs that are domain testable have no input-output inconsistencies, so in some sense, they are are "easily testable." Domain testability is defined in terms of the properties of observability and controllability. Informally, a software component is observable if distinct outputs are observed for distinct inputs: observability is the ease of determining if specified inputs affect the outputs. A software component is controllable if given any desired output value, an input exists that "forces" the component output to that value: controllability is the ease of producing a specified output from a specified input.

Observability and controllability were first developed for dynamical systems and automata [17]. Dynamical systems have inputs, outputs, states, and state transition functions. For hardware components, the state transition functions are more easily identifiable than the state transition functions for software components. Controllability and observability properties have been used for specifying hardware testability [19]. 


\section{Testability of Software Components}

Our initial focus is in specifying the observability and controllability properties for two software components: expression procedures (similar to Ada function subprograms) and command procedures (similar to Ada procedure subprograms). The domain testability properties of these components are defined and generalized in Section II. We define metrics for practically comparing observability and controllability. The properties and metrics are illustrated In Section III. In Section IV, we discuss how testability can be assessed from program specifications, and discuss an experiment that shows that it takes less time to build and test a program developed from a domain testable specification than a similar program developed from a non-domain testable specification. In Section V, other aspects of software observability and controllability are suggested for future work.

We show in the Appendix how software inputs, outputs, and state transitions can be represented with the direct execution models of denotational semantics $[13,24,26]$ to yield a more formal definition of observability and controllability.

\section{Domain Testability}

Domain testability is defined in terms of the execution semantics of program inputs and outputs. Our model programming language has the syntax of an Ada-like language: a more formal definition of domain testability in terms of denotational semantics is specified in the Appendix.

Domain testability is defined by specifying the semantics of expression evaluation and command execution. Expressions are denoted by functions (also called expression procedures). For example, the following function

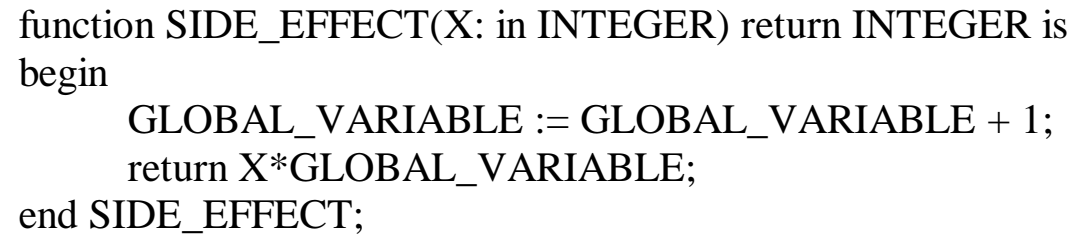

shows that the evaluation of an expression depends on the state, and results in a value and a new state.

Commands are denoted by commands (also called command procedures). For example, the following command procedure 
Testability of Software Components

procedure SIDE_EFFECT(X: in INTEGER; Y: out INTEGER) is begin

GLOBAL_VARIABLE :=GLOBAL_VARIABLE + 1;

$\mathrm{Y}:=\mathrm{X} * \mathrm{GLOBAL}$ VARIABLE;

end SIDE_EFFECT;

shows that execution depends on the state, and results in a new state.

\section{A. Observability}

An expression procedure $\mathrm{F}$ is observable if distinct outputs are generated from distinct inputs. Since the evaluation of the output of $\mathrm{F}$ is a function of the evaluation of its input and the state, observability implies that the output value of $\mathrm{F}$ is a function of the input value only.

For example, the following expression procedure is not observable:

function $\mathrm{F}(\mathrm{X}$ : in INTEGER) return INTEGER is

begin

return $X^{*}$ GLOBAL_VARIABLE;

end F;

The reason that it is not observable is that subsequent calls to the function $\mathrm{F}$ with the same arguments can yield different results. In an environment where GLOBAL_VARIABLE evaluates to 0 , the function evaluation $\mathrm{F}(3)$ evaluates to 0 . If the environment is changed after this function call where GLOBAL_VARIABLE $=2$, then $\mathrm{F}(3)=$ evaluates to 6 .

In general, given an expression procedure $\mathrm{F}$ :

function $\mathrm{F}(\mathrm{E} 1$ : in $\mathrm{T} 1 ; \mathrm{E} 2$ : in $\mathrm{T} 2 ; \ldots$; En: in Tn) return TF;

$\mathrm{F}$ is observable if

$$
\mathrm{F}(\mathrm{B} 1, \ldots, \mathrm{Bn}) ? \mathrm{~F}(\mathrm{~A} 1, \ldots, \mathrm{An}) \text { implies }(\mathrm{B} 1, \ldots, \mathrm{Bn}) ?(\mathrm{~A} 1, \ldots, \mathrm{An}) .
$$

An execution of a command procedure is observable if distinct outputs are generated from distinct inputs. For example, the following command procedure is not observable:

procedure $\mathrm{C}(\mathrm{X}$ : in INTEGER; $\mathrm{Y}$ : out INTEGER) is

begin

$\mathrm{Y}:=\mathrm{X}^{*} \mathrm{GLOBAL}$ VARIABLE;

end $\mathrm{C}$; 


\section{Testability of Software Components}

The reason that it is not observable is that subsequent calls to the procedure with the same arguments can yield different results. In an environment where GLOBAL_VARIABLE $=0$, $\mathrm{C}(3, \mathrm{Y})$ yields $\mathrm{Y}=0$. If the environment is changed after this function call where GLOBAL_VARIABLE $=2$, then $\mathrm{Y}=6$.

In general, given a command procedure $\mathrm{P}$ :

procedure $\mathrm{P}(\mathrm{E} 1$ : in $\mathrm{S} 1 ;$...En: in $\mathrm{Sn} ; \mathrm{V} 1$ : in $\mathrm{T} 1 ; \ldots \mathrm{Vm}$ : out $\mathrm{Tm})$;

$\mathrm{P}$ is observable if

$$
(\mathrm{Y} 1, \ldots, \mathrm{Ym}) ?(\mathrm{Z} 1, \ldots, \mathrm{Zm}) \text { implies }(\mathrm{X} 1, \ldots, \mathrm{Xn}) ?(\mathrm{U} 1, \ldots, \mathrm{Un})
$$

given the executions

and

$$
\mathrm{P}(\mathrm{X} 1, \ldots, \mathrm{Xn}, \mathrm{Y} 1, \ldots, \mathrm{Ym})
$$

$\mathrm{P}(\mathrm{U} 1, \ldots, \mathrm{Un}, \mathrm{Z1}, \ldots, \mathrm{Zm})$;

for all $(\mathrm{X} 1, \ldots, \mathrm{Xn})$ and $(\mathrm{U} 1, \ldots, \mathrm{Un})$ in domains $(\mathrm{S} 1, \ldots, \mathrm{Sn})$.

\section{B. Controllability}

Given a state s, the domain of values of the evaluation for expressions is usually a proper subset of the target type. An evaluation of expression procedure $\mathrm{F}(\mathrm{E})$ is controllable if, for any state $\mathrm{s}$, the domain of values of the evaluation map equals the domain of values denoted by its output specification.

For example, in an environment where type POSITIVE denotes the set of values $\{0,1,2, \ldots$, MAX_INTEGER $\}$, and given the expression procedure $G$

function $\mathrm{G}(\mathrm{X}$ : in POSITIVE) return POSITIVE is

begin return $\mathrm{X} \bmod 3$;

end;

$\mathrm{G}$ is observable, because if $\mathrm{G}(\mathrm{A} 1)=\mathrm{b} 1$ and $\mathrm{G}(\mathrm{A} 1)=\mathrm{b} 2$ then $\mathrm{b} 1=\mathrm{b} 2$. However, the expression procedure $\mathrm{G}$ is not controllable. Even though $\mathrm{G}$ returns a subset of type POSITIVE, for every input (the set $\{0,1,2\}$ ), there is no set of inputs that evaluates to POSITIVE: $\{0,1,2\}$ ?

POSITIVE.

If expression procedure $\mathrm{F}$ 


\section{Testability of Software Components}

function $\mathrm{F}(\mathrm{E} 1$ : in $\mathrm{T} 1 ; \mathrm{E} 2:$ in $\mathrm{T} 2 ; . .$. ; En: in Tn) return TF;

is controllable, then for for all input values of $\mathrm{A} 1, \ldots, \mathrm{An}$

$$
\{\text { all evaluations of } \mathrm{F}(\mathrm{A} 1, \ldots, \mathrm{An})\}=\mathrm{TF}
$$

If $\mathrm{F}$ is observable and controllable, them $\mathrm{F}$ is an onto function.

Observability is not required for controllability: programming languages that define functions having no input variables may be controllable but not observable. For example, given

\section{function RANDOM() return INTEGER;}

If the set of all evaluations of RANDOM() covers all values in INTEGER, then RANDOM is controllable.

An execution of a command procedure is controllable if, for any state s, the domain of each output value equals its type. Given command procedure $\mathrm{P}$

procedure $\mathrm{P}(\mathrm{E} 1$ : in $\mathrm{S} 1 ;$...En: in $\mathrm{Sn} ; \mathrm{V} 1$ : in $\mathrm{T} 1 ; \ldots \mathrm{Vm}$ : out $\mathrm{Tm})$;

$\mathrm{P}$ is controllable if

$$
\{(\mathrm{Y} 1, \ldots, \mathrm{Ym})\}=(\mathrm{T} 1, \ldots, \mathrm{Tm})
$$

given all executions

$$
\mathrm{P}(\mathrm{X} 1, \ldots, \mathrm{Xn}, \mathrm{Y} 1, \ldots, \mathrm{Ym})
$$

for all $(\mathrm{X} 1, \ldots, \mathrm{Xn})$ in $(\mathrm{S} 1, \ldots, \mathrm{Sn})$.

\section{Domain Testability}

Since testing assesses the compliance of a program to intended input-output specifications, it is necessary to identify the data characteristics of the explicit test inputs and outputs (inferred from specific identifiers in the program) and implicit test inputs and outputs (inferred from component states -- not from specific identifiers). Part of the difficulty with testing is concerned with identifying and specifying implicit program inputs and outputs from states as auxiliary inputs and outputs that can be controlled and observed, so that test results do not exhibit the input-output inconsistencies defined in Section I. 


\section{Testability of Software Components}

The definitions of observability and controllability immediately establish the following:

Corollary: If an expression (command) procedure is not observable, then its evaluation (execution) may exhibit output inconsistencies. If an expression (command) procedure is not controllable, then its evaluation (execution) can exhibit input inconsistencies.

Definition: An expression (command) procedure is domain testable if it is observable and controllable.

Programs that are not domain testable can be difficult to test. For example, the following is a specification for a common expression procedure that returns a random floating point number between zero and one according to a uniform probability distribution:

\section{function RANDOM(): return REAL;}

This function is not observable since it has no input (in fact, it is not supposed to be observable by design). It is desired to be controllable. Testing this function is difficult without any additional knowledge about its internal states.

Most function and expression procedures are not a priori observable or controllable. This means that without any further modification, effective and adequate testing is difficult to assess: there may be input or output inconsistencies. In order to test, many software engineers modify the program by explicitly creating additional program inputs and outputs that denote the implicit program states. This improves testability by making these states observable and controllable: the program can then be input to a test driver. One problem with this modification is that the tested component may be different from the actual deployed component.

We formalize this program modification process with the following

Definition: Domain testability refers to the ease of modifying a program so that it is observable and controllable.

The program modifications required to achieve domain testability are called extensions. Observable extensions introduce program inputs based on implicit states; controllable extensions modify outputs.

\section{Observable Extensions}

Let $\mathrm{F}$ denote the expression procedure definition function $\mathrm{F}(\mathrm{E}$ : in $\mathrm{T})$ return $\mathrm{TF}$; 


\section{Testability of Software Components}

$\mathrm{F}$ has an observable extension with observability index $\mathrm{n}$ if there exists an observable expression procedure FO, with definition

function $\mathrm{FO}(\mathrm{E}$ : in $\mathrm{T}$; E1: in T1; E2: in T2; .. ; En: in Tn) return TF;

such that for all inputs of $\mathrm{F}$, there exists inputs for FO such that

$$
\mathrm{F}(\mathrm{E})=\mathrm{FO}(\mathrm{E}, \mathrm{E} 1, \mathrm{E} 2, \ldots, \mathrm{En})
$$

For example, the following expression procedure $\mathrm{F}$

function $\mathrm{F}(\mathrm{X}$ : in INTEGER) return INTEGER is

begin return $X^{*}$ GLOBAL_VARIABLE;

end $\mathrm{F}$;

has an observable extension FO:

function FO (X: in INTEGER; G: in INTEGER) return INTEGER is begin return $\mathrm{X}^{*} \mathrm{G}$;

end FO;

$\mathrm{FO}$ is observable, since for given inputs $(\mathrm{A} 1, \mathrm{~A} 2)$ and $(\mathrm{B} 1, \mathrm{~B} 2), \mathrm{F}(\mathrm{A} 1, \mathrm{~A} 2)$ ? $\mathrm{F}(\mathrm{B} 1, \mathrm{~B} 2)$ implies $(\mathrm{A} 1, \mathrm{~A} 2)$ ? (B1,B2).

A command procedure $\mathrm{P}$

$$
\text { procedure } \mathrm{P}(\mathrm{E} \text { : in } \mathrm{T} \text {; } \mathrm{O} \text { : out } \mathrm{TP}) \text {; }
$$

has an observable extension with observability index $\mathrm{n}$ if there exists an observable command procedure $\mathrm{PO}$, with definition

procedure PO (E: in T; E1: in S1; ...En: in Sn; O: out TP; OE: TE);

such that for all inputs for $\mathrm{P}$, there exists a set of inputs for PO such that after the execution of

and

$$
\mathrm{P}(\mathrm{E}, \mathrm{O})
$$

$$
\text { PO }(\mathrm{E}, \mathrm{E} 1, \ldots, \mathrm{En}, \mathrm{OE})
$$

then $\quad \mathrm{O}=\mathrm{OE}$. 
For example, command procedure $\mathrm{C}$

procedure $\mathrm{C}(\mathrm{X}$ : in INTEGER; $\mathrm{Y}$ : out INTEGER) is

begin

end $\mathrm{C}$;

$$
\mathrm{Y}:=\mathrm{X} * \mathrm{GLOBAL} \text { _VARIABLE; }
$$

has is an observable extension of command procedure $\mathrm{CO}$ :

procedure CO (X: in INTEGER; G: in INTEGER; Y: out INTEGER) is begin $\mathrm{Y}:=\mathrm{X}^{*} \mathrm{G}$ end $\mathrm{CO}$;

\section{E. Controllable Extensions}

Given

function $\mathrm{F}(\mathrm{E} 1$ : in $\mathrm{T} 1 ; \ldots$; En: in Tn) return TF;

F has an controllable extension if there exists a type TC that is a subset of TF and a controllable expression function $\mathrm{FC}$, with definition function $\mathrm{FC}(\mathrm{E}$ : in T ; E1: in T1; E2: in T2; ... ; En: in Tn) return TC;

such that for all inputs and any state s for F, there exists a state $\mathrm{s}^{*}$ for FC such that

$$
\mathrm{F}(\mathrm{E} 1, \ldots, \mathrm{En})=\mathrm{FC}(\mathrm{E} 1, \ldots, \mathrm{En})
$$

For example, the following expression procedure GC is a controllable extension of $\mathrm{G}$ (defined in Section II.C):

type SMALL is POSITIVE range 0..2;

function GC (X: in POSITIVE) return SMALL is

begin

return SMALL'(X mod 3);

end GC;

GC is controllable, since the set of values it returns is the same set denoted by the type SMALL:

$\{\mathrm{GC}(\mathrm{E})$ for all $\mathrm{E}\}=\{0,1,2\}=\mathrm{SMALL}$ 
Moreover, for all states s for $\mathrm{G}$, there exists an s* for $\mathrm{GC}$ so that

$$
\mathrm{G}(\mathrm{E})=\mathrm{GC}(\mathrm{E})
$$

A command procedure $\mathrm{P}$

$$
\text { procedure } \mathrm{P}(\mathrm{E} \text { : in } \mathrm{T} ; \mathrm{O} 1 \text { : out } \mathrm{S} 1 ; \ldots \text {; Om: out } \mathrm{Sm}) \text {; }
$$

has a controllable extension with controllability index $\mathrm{m}$ if there exists a types $\mathrm{T} 1, \ldots, \mathrm{Tm}$ that are a subset of $\mathrm{S} 1, \ldots, \mathrm{Sm}$ respectfully, and a controllable command procedure $\mathrm{PC}$, with definition

procedure PC (E: in T; OC1: out $\mathrm{T} 1 ; \ldots$; OCm: out Tm);

such that for all inputs and any state s for P, there exists a state s* for PC such that

$$
(\mathrm{O} 1, \ldots, \mathrm{Om})=(\mathrm{OC} 1, \ldots \mathrm{OCm})
$$

Given the executions

and

$$
\mathrm{P}(\mathrm{A}, \mathrm{B} 1, \ldots \mathrm{Bm})
$$

$$
\mathrm{PC}(\mathrm{A}, \mathrm{BC} 1, \ldots \mathrm{BCm})
$$

Controllable extensions depend on the richness of type domain definitions. For conventional languages, these definitions may be modeled by inductive assertions [10]. For functional languages, type domains are function domains [1].

\section{F. Measures of Domain Testability}

We formally define domain testability measures in terms of controllable and observable extensions.

Observability is the ease of determining if specified inputs effect the outputs; Controllability ease of producing a specified output from a specified input. 


\section{Testability of Software Components}

Given the following expression procedure

function $\mathrm{F}(\mathrm{E}$ : in $\mathrm{T})$ return $\mathrm{TF}$;

and observable extension with observability index n:

function $\mathrm{FO}(\mathrm{E}:$ in $\mathrm{T} ; \mathrm{E} 1$ : in $\mathrm{T} 1 ; \mathrm{E} 2:$ in $\mathrm{T} 2 ; \ldots$; En: in $\mathrm{Tn})$ return $\mathrm{TF}$;

For an exhaustive test, the extra number of test cases required would be a multiple of

$$
|\mathrm{T} 1|^{*} \ldots *|\mathrm{Tn}|=\left|\mathrm{T}_{\max }\right|^{\mathrm{n}}
$$

Here, $|\mathrm{Tk}|$ denotes the cardinality of the domain $\mathrm{Tk}$, and $\left|\mathrm{T}_{\max }\right|$ is the maximum cardinality of the domain over all input types. Even though this value can be infinite for dynamic data structures such as strings and lists, during testing we enforce pragmatic limits to dynamic data structures.

The extra testing work associated with the n extra inputs depend on the type; we can normalize this factor by considering the effective number of extra binary inputs:

$$
\mathrm{Ob}=\log _{2}\left(|\mathrm{~T} 1|^{*} \ldots *|\mathrm{Tn}|\right)
$$

$\mathrm{Ob}$ is the measure of observability and corresponds to the number of extra binary inputs required to make a program observable. The measure of observability for command procedures is similar.

Given the command procedure $\mathrm{P}$

$$
\text { procedure } \mathrm{P}(\mathrm{E} \text { : in } \mathrm{T} ; \mathrm{O} 1 \text { : out } \mathrm{S} 1 ; \ldots \text {; Om: out } \mathrm{Sm}) \text {; }
$$

and controllable extension with controllability index m

$$
\text { procedure PC (E: in T; OC1: out } \mathrm{T} 1 ; \ldots \text {; OCm: out } \mathrm{Tm}) \text {; }
$$

We define

$$
\mathrm{Ct}=\log _{2}\left(|\mathrm{~T} 1|^{*} \ldots *|\mathrm{Tm}|\right)
$$

$\mathrm{Ct}$ is the measure of observability and corresponds to the number of binary inputs that must be modified to make a program controllable.

We note that

$$
0=\mathrm{Ob}=\mathrm{n} * \log _{2}\left(\left|\mathrm{~T}_{\max }\right|\right)
$$




$$
0=\mathrm{Ct}=\mathrm{m} * \log _{2}\left(\left|\mathrm{~T}_{\max }\right|\right)
$$

These last two expressions are similar in form to the software science volume complexity metrics [25]. The lower bounds are obtained if the program is observable or controllable.

For imperative programming languages, we have the following results that allow us to easily compute $\mathrm{Ob}$ and $\mathrm{Ct}$ so that they can be compared. These results follow from the definition of the logarithm and the combinatorial (multiplicative) nature of additional testing inputs.

\section{Sequence}

The measures of observability and controllability of a sequence (composition) of commands (expressions) is the sum of the measures of the components:

In other words

$$
\begin{aligned}
& \mathrm{Ob}(\mathrm{C} 1 ; \mathrm{C} 2)=\mathrm{Ob}(\mathrm{C} 1)+\mathrm{Ob}(\mathrm{C} 2) \\
& \mathrm{Ct}(\mathrm{C} 1 ; \mathrm{C} 2)=\mathrm{Ct}(\mathrm{C} 1)+\mathrm{Ct}(\mathrm{C} 2)
\end{aligned}
$$

\section{Selection}

Given an observable predicate $\mathrm{E}$, the measures of observability and controllability of a selection of commands is a function of the measures of the components:

In other words

$$
\begin{aligned}
& \mathrm{Ob} \text { (if } \mathrm{E} \text { then } \mathrm{C} 1 \text {; else } \mathrm{C} 2 \text { end if; })=\mathrm{Ob}(\mathrm{C} 1)+\mathrm{Ob}(\mathrm{C} 2) \\
& \mathrm{Ct} \text { (if } \mathrm{E} \text { then } \mathrm{C} 1 \text {; else } \mathrm{C} 2 \text { end if; })=\mathrm{Ct}(\mathrm{C} 1)+\mathrm{Ct}(\mathrm{C} 2)+\mathrm{Ct}(\mathrm{E})
\end{aligned}
$$

\section{Iteration}

Given an observable predicate $\mathrm{E}$, the measures of observability and controllability of the iteration of a command is a function of the measures of the components:

In other words

$\mathrm{Ob}$ (while E loop C1; end loop; $)=\mathrm{Ob}(\mathrm{C} 1)$

$\mathrm{Ct}$ (while E loop $\mathrm{C} 1$; end loop; $)=\mathrm{Ct}(\mathrm{C} 1)+\mathrm{Ct}(\mathrm{E})$

\section{Examples}

\section{A. Text Formatter}


Naur [22] investigated the application of formal program proving in the construction of a simple formatting program. This program was subsequently tested and analyzed by Goodenough [11]. Meyers [21] translated the program to PL/I and had it tested by a number of professional programmers. Basili[2] also used this program in a similar study of test effectiveness. In a review of the Meyers study, House [15] stated that the program was virtually untestable since it was so poorly specified.

Part of the problem was in its reliance on the side-effects of input and output. For example, the PL/I output character procedure is shown in Figure 2.

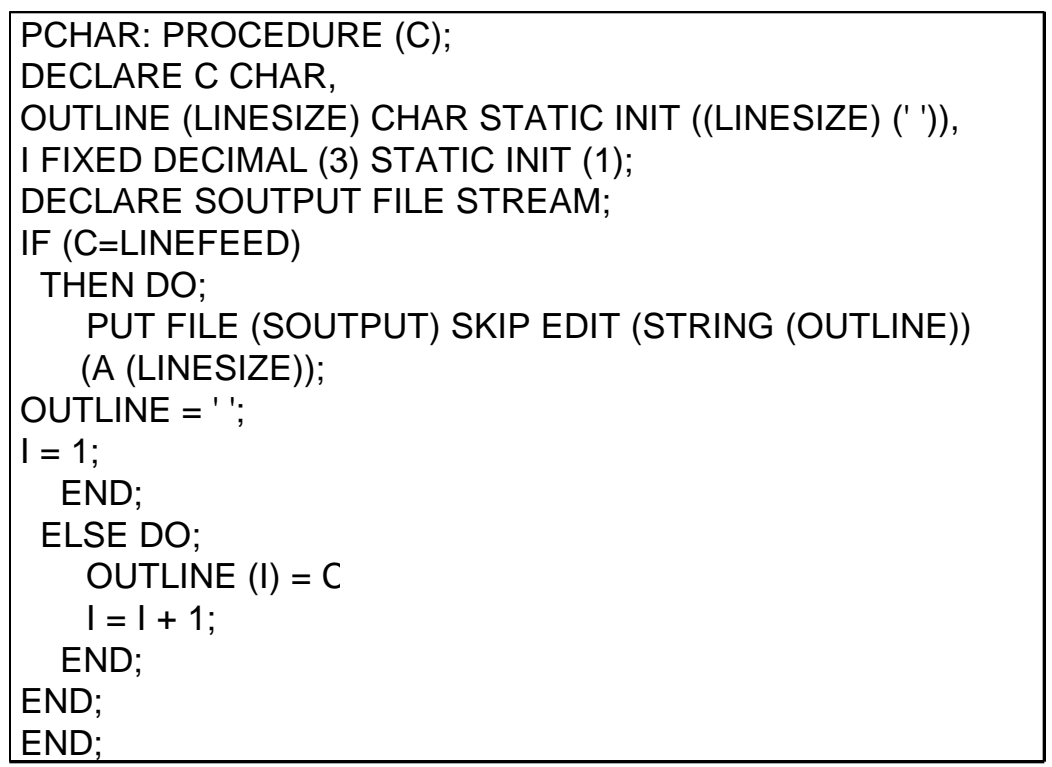

Figure 2. Output Character Procedure for Formatting Example

This procedure has the side-effects (among others) of writing a character to the output stream, changing an implicit column number, line number, or possibly a page number. If the command results include text output displays, subsequent executions of $\operatorname{PCHAR}(\mathrm{X})$ result in different results, so PCHAR is not observable. In order to test it, an observable and controllable extension such as

procedure PCHART(COLUMN: in COLUMN_TYPE;

LINE: in LINE_TYPE;

PAGE: in PAGE_TYPE;

FILE: in FILE_TYPE

ITEM: in CHARACTER;

STRING: out STREAM); 


\section{Testability of Software Components}

can be defined so that the execution of this command would yield the same result for equal arguments. The execution of this procedure will write all literal "printable" characters to a specified output stream. In this example, the observability index is 4 and the controllability index is 1 . Given the following domain cardinalities for the test

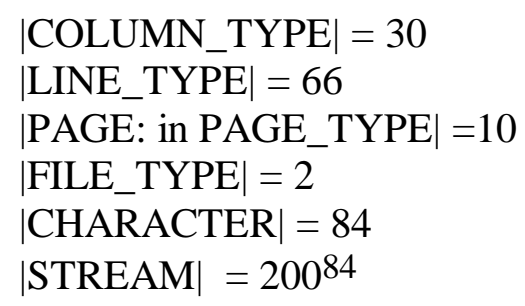

The STREAM cardinality assumes that a stream is 200 characters. The observability measure is 21.7 and the controllability measure is 642 .

\section{B. Calendar Program}

This program, initially proved correct, was later discovered to have several errors. This program is discussed in Geller[10] and Lamport[18]. The program is specified in ALGOL-W in Figure 3.

The procedure is not observable since there is no output. It is also not controllable: for example, in the procedure, day1 is allowed be negative; allowing negative-valued days indicates an error in the specification.

An observable and controllable extension can be given as:

$$
\begin{aligned}
\text { procedure calendart (day1, day2 in : DAY_TYPE; } \\
\text { month1, month2 in MONTH_TYPE; } \\
\text { year: in YEAR_TYPE; } \\
\text { days_between: out POSITIVE_INTEGER); }
\end{aligned}
$$

This modification required five new inputs and one new output. Given the following domain cardinalities for a test:

$$
\begin{aligned}
& \mid \text { DAY_TYPE } \mid=31 \\
& \mid \text { MONTH_TYPE } \mid=12 \\
& \mid \text { YEAR_TYPE } \mid=300 \\
& \mid \text { POSITIVE_INTEGER } \mid=2^{31}
\end{aligned}
$$

The observability measure is 25.3 and the controllability measure is 31 . 


\section{Testability of Software Components}

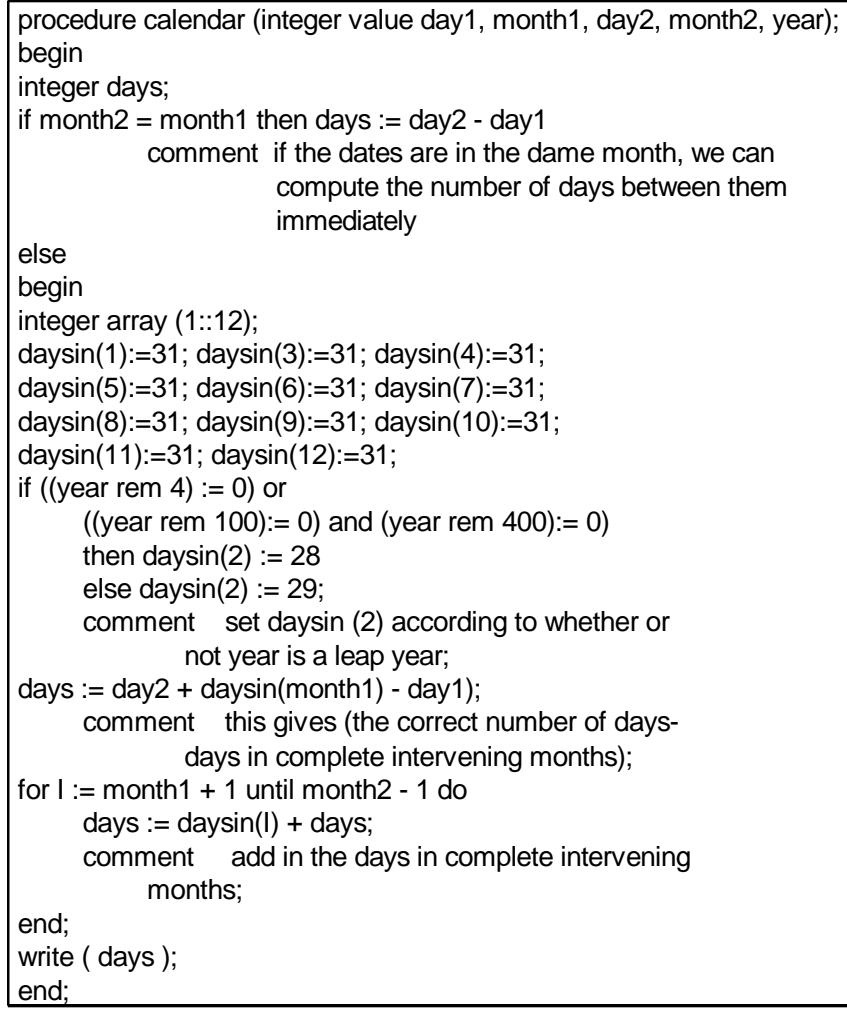

Figure 3. The Calendar Program

\section{91 Function}

This function was also discussed by Geller in [10] as an example of a program that was proved correct. The function returns the number 91 when $X=101$ or returns $X-10$ when $X>100$. Its full specification is

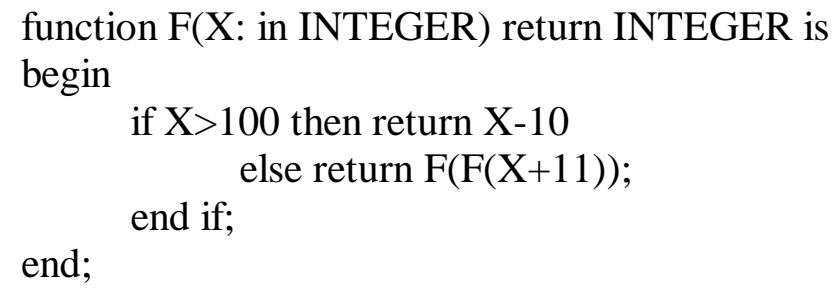

The function is observable. It is not controllable since it does not return all integers. A controllable extension can be defined with the auxiliary type

type RETURN_TYPE is new INTEGER range 91..MAX_INT; 


\section{Testability of Software Components}

If MAX_INT has a value of $2^{31}$ then the controllability measure is

$$
\log _{2}\left(2^{31}-90\right)=30.99
$$

\section{Concurrent Tasks}

This example demonstrates a common testing problem seen in transaction processing. The testing anomaly is discussed in Eswaren et al[4], and Gardarin and Valduriez [9]. In Ada-like syntax, we have

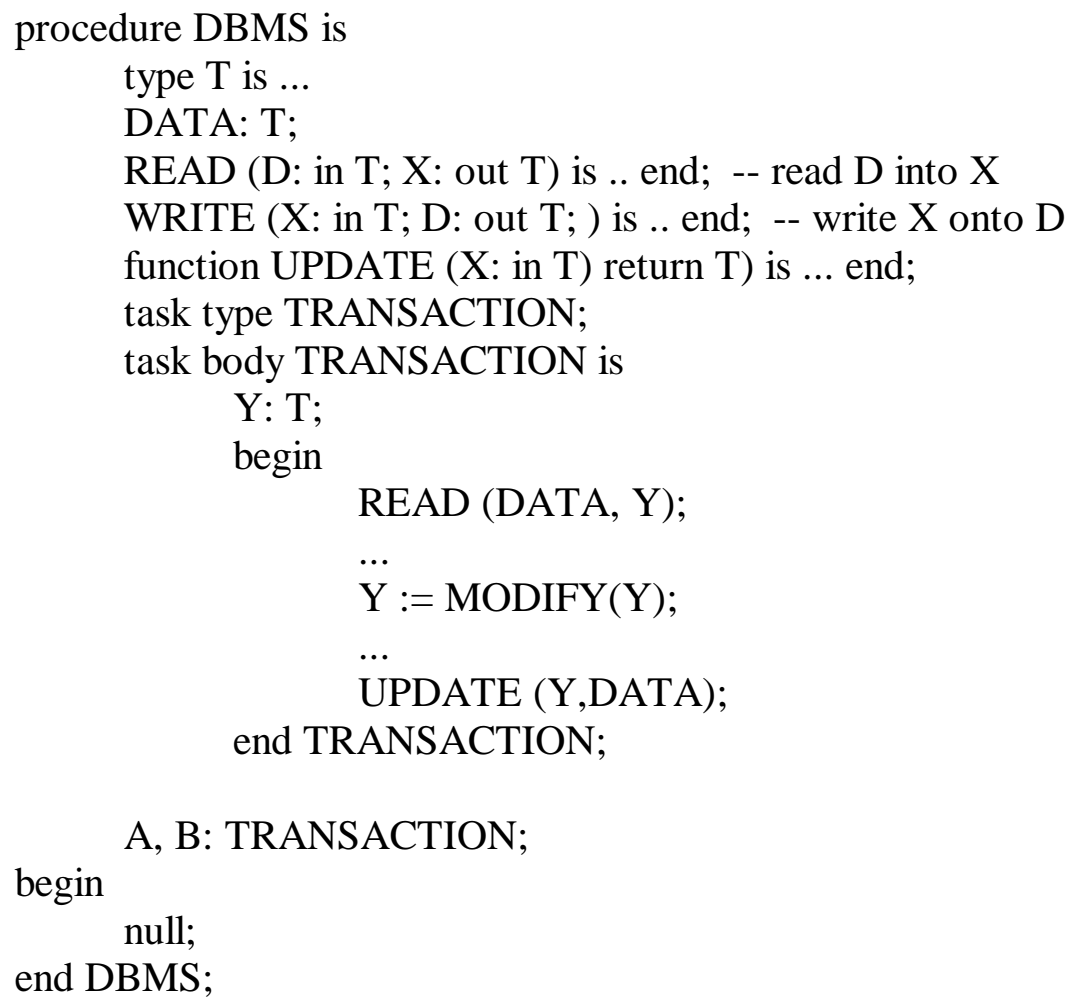

If READ and UPDATE are observable and controllable in a sequential execution environment, they are not observable and controllable in a concurrent execution environment because of the side-effects due to the scheduling of operations. For example, suppose while task A starts reading DATA, task B has already read and updated DATA. Any subsequent reference to DATA by A yields different results. Consequently, READ is not observable. Moreover, any updates made by $\mathrm{B}$ are lost. Consequently, UPDATE is not controllable. Procedures READ and WRITE have observable and controllable extensions if the tasks can be provided with serializable schedules [9]: read and updating must be encapsulated so that these operations appear as if executing 


\section{Testability of Software Components}

sequentially. Observable and controllable extensions may introduce the states GLOBAL_TIME and LOCK? as extra input and outputs.

\section{Discussion}

\section{A. Software Requirements, Specifications, and Domain Testability}

A key principle of software engineering is that one of the best ways to prevent errors and to improve the effectiveness of testing is by having a good specification and a good set of functional requirements.

Knowledge of module inputs, outputs, and states do not require executable programs: they should be stated in a functional requirement or design specification. Consequently, testability can be assessed from program specifications. By incorporating the ideas of observability and controllability into the requirement and specification process, hidden program states are made explicit in the specification.

This means that the testing task of "identifying the input and output, states, and state transitions," and modifying the program so that these characteristics are observable and controllable is simplified.

By building software from domain testable specifications, software builders do not need to waste time on input and output inconsistencies and the resultant program modifications. A domain testable program reduce the redundancy of test sets (and consequently, the amount of testing). The test results are also more readable, since implicit states are made explicit.

\section{B. Advantages of Domain Testability: An Experiment}

As observed above, programs developed from domain testable specifications should be "easier" to test. The following experiment shows that it takes less time to build and test a program developed from a domain testable specification than a similar program developed from a nondomain testable specification.

Eight undergraduate students were asked to build and test two Text Formatting programs in a language of their choice. They were given two references on the Text Formatter program, [15] and [21] to help them develop their specifications.

For the first program, all students were asked to use the Meyers PL/I program as a specification and translate the PL/I program into the language of their choice. As observed in Section III.A, this PL/I program contains components that are neither observable and controllable. They were then asked to test the translated program to determine if their translation uncovered any of the 15 errors documented in [21]. They were not required to debug the program. The students recorded the number of hours required for this build-and-test activity. 


\section{Testability of Software Components}

The second program involved a total redesign of the Text Formatter. The students were split into two groups. Each student in Group A developed a domain testable specification of the Text Formatter: their functions and procedures were specified to be domain testable as indicated in Section III.A. Each student in Group B did not develop a domain testable specification. They were then asked to test the redesigned program to determine if their program uncovered any of the 15 errors documented in [21]. The students also recorded the number of hours required for this build-and-test activity.

Our hypothesis is that there is a measurable difference in time for the build-and-test activities between domain testable and non-domain testable specifications.

The results of the experiment are in Figure 4.

\begin{tabular}{|c|c|c|c|}
\hline Student & $\begin{array}{c}\text { Translation } \\
\text { Time }\end{array}$ & $\begin{array}{l}\text { Redesign } \\
\text { Time }\end{array}$ & $\begin{array}{l}\text { Redesign Time } \\
\text { Translation Time }\end{array}$ \\
\hline Rivera & 4 & 2.5 & 0.625 \\
\hline Hendrie & 8 & 5 & 0.625 \\
\hline Finegan & 8 & 5 & 0.625 \\
\hline Huang & 11 & 9 & 0.818181818 \\
\hline Aoki & 13 & 11 & 0.846153846 \\
\hline Ip & 5 & 4.75 & 0.95 \\
\hline Chieu & 10 & 10 & 1 \\
\hline Kornish & 7.5 & 10.5 & 1.4 \\
\hline Average & 8.3125 & 7.21875 & 0.861166958 \\
\hline Std. Deviation & 2.99 & 3.05 & 0.26 \\
\hline
\end{tabular}

Figure 4. Text Formatter Experiment: Results.

As expected, there are large variances in reported development time. One reason for these variances may be due to a student bias in reporting times. In order to eliminate this bias, we compute a normalizing ratio

$$
\mathrm{R}=\frac{\text { Redesign Time }}{\text { Translation Time }}
$$

which provides a normalized measure of productivity for each individual student.

In this experiment, the average value of $\mathrm{R}$ is 0.87 ; this means that the time required to develop a "testable" program from the original PL/I specification was, in most cases, at least as great as the time required to develop a redesigned program. This was noted informally in [15]. 
When we display the results in Figure 4 by Group, we see the advantages of a domain testable specification. This is seen in Figure 5 for Group A and Figure 6 for Group B.

\begin{tabular}{l|l|c|c|}
\hline \multicolumn{1}{|c}{$\begin{array}{c}\text { Translation } \\
\text { Time }\end{array}$} & \multicolumn{1}{c}{$\begin{array}{c}\text { Redesign } \\
\text { Time }\end{array}$} & $\begin{array}{r}\text { Redesign Time } \\
\text { Translation Time }\end{array}$ \\
\hline Aoki & 13 & 11 & 0.846153846 \\
Finegan & 8 & 5 & 0.625 \\
Huang & 11 & 9 & 0.818181818 \\
Rivera & 4 & 2.5 & 0.625 \\
\hline \multicolumn{4}{|c}{} \\
Average & 9 & 6.875 & 0.728583916 \\
Std. Deviation & 3.92 & 3.84 & 0.12 \\
\hline
\end{tabular}

Figure 5. Text Formatter Experiment: Group A Results.

\begin{tabular}{|l|c|c|c|}
\hline \multicolumn{1}{|c}{} & \multicolumn{1}{c}{$\begin{array}{c}\text { Translation } \\
\text { Time }\end{array}$} & \multicolumn{1}{c}{$\begin{array}{c}\text { Redesign } \\
\text { Time }\end{array}$} & $\begin{array}{r}\text { Redesign Time } \\
\text { Translation Time }\end{array}$ \\
\hline Ip & 5 & 4.75 & 0.95 \\
Kornish & 7.5 & 10.5 & 1.4 \\
Chieu & 10 & 10 & 1 \\
Hendrie & 8 & 5 & 0.625 \\
\hline \multicolumn{4}{|c}{} \\
Average & 7.625 & 7.5625 & 0.99375 \\
Std. Deviation & 2.06 & 3.11 & 0.32 \\
\hline
\end{tabular}

Figure 6. Text Formatter Experiment: Group B Results.

We see that the average value of $\mathrm{R}$ for Group $\mathrm{A}$ is 0.72 and the average value of $\mathrm{R}$ for Group $\mathrm{B}$ is 0.99. This indicates that it takes on the average $27 \%$ less time to build and test a program developed from a domain testable specification than it takes to build and test a similar program developed from a non-domain testable specification.

\section{Applying the Measures of Observability and Controllability}

The observability and controllability measures of a domain testable component are zero. For components that are not domain testable, the observability and controllability measures can 


\section{Testability of Software Components}

provide a practical normalized metric for assessing the work involved in modifying a program to be domain testable. It is important to note that these metrics indicate testing effort only so far as the effort required to modify a component to a domain testable form.

The observability and controllability measures can also be used to develop a normalized metric for for assessing the work involved in modifying a system to be domain testable: according to the properties in Section II.C., observability and controllability increase linearly with the number of components.

The metrics can be calibrated by building a database of program components coupled with the time used to make these components domain testable. In this way, the metrics can be used indirectly to assess testing time. 


\section{Conclusions and Future Work}

A computer program can be modeled as a complex state machine that transforms inputs to outputs. In a liberal reading of the second law of thermodynamics, since a "perfect" machine does not exist, it follows that a "perfect" computer program does not exist either. The goal of testing is less demanding than the goal of producing a "perfect" program: the testing goal is to determine the likelihood of a program having errors. This goal can be met if we understand the meaning of software testability, as well as software computability.

The significant contribution of this paper was to formally investigate the meaning of software testability. We defined a new concept, domain testability, by applying the concepts of observability and controllability to programs. We showed that a domain testable program does not exhibit any input-output inconsistencies. Consequently, domain testable programs will support small test sets, where test outputs are easily understood.

We also defined new metrics that can be used to assess the level of effort required in order to modify a program so that it is domain testable.

Several other areas suggest themselves for future research.

Domain Testability for other programming structures. Can observability and controllability be defined for programming structures that are not restricted to commands or expressions, like definitions, generic units and objects? Are the denotational methods described in the Appendix applicable?

Observability and continuity. Watkins [28] discusses a testing technique based on a predictorcorrector strategy that is based on the continuity properties of a real-valued expression procedure. Can observability be extended to include a topology defined on an input domain, so that the output is a continuous function of the input?

Domain testability and functional dependencies. Is there a relationship between the transitive functional dependencies (used, for example, in normalizing databases [9]) and the functional dependencies between inputs and outputs of an observable component?

\section{Acknowledgements:}

I wish to thank wish to thank Eric J. Finegan for helping me revise the paper, and the very helpful suggestions of the reviewers.

\section{References}




\section{Testability of Software Components}

[1] J. Backus, "Can Programming Be Liberated from the von Neumann Style? A Functional Style and its Algebra of Programs," Comm. ACM, vol 21, no. 8, pp. 613-641, August, 1978.

[2] Basili, V., and Selby, R.W., "Comparing the Effectiveness of Software Testing Strategies," IEEE Trans. Software Eng., vol. SE-13, pp. 1278-1296, December, 1987.

[3] B. Beizer, Software Testing Techniques,. New York:Van Nostrand Reinhold Co, 1983.

[4] K.P. Eswaran, J.N. Gray, R.A. Lorie, L.L. Traiger, "The Notion of Consistency and Predicate Locks in a Database System", Comm. ACM, vol. 19, no. 11, pp. 624-633, November 1976.

[5] R. Freedman, Programming with APSE Software Tools. Petrocelli Books: Princeton, 1985.

[6] R. Freedman, M. Shooman, M. Corcoran, R. Frail, "A Knowledge-Based Testing Assistant," Polytechnic Notes on Artificial Intelligence, no. 5, Polytechnic University, 1987.

[7] R. Freedman, M. Shooman, M. Corcoran, R. Frail, "An Expert System for Software Component Testing," University Seminar on Artificial Intelligence, Unisys Corporation, Nice (France), October 1987.

[8] H. Fujiwara, Logic Testing and Design for Testability. Cambridge: MIT Press, 1985.

[9] G. Gardarin and P. Valduriez, Relational Databases and Knowledge Bases. New York: Addison Wesley, 1989.

[10] M. Geller, Test data as an aid in proving program correctness. Comm. ACM, vol. 21, no. 5, pp. 368-375, May, 1978.

[11] J. B. Goodenough and S. L. Gerhart, Toward a Theory of Test Data Selection," IEEE Trans. Software Eng., vol. SE-1, pp. 156-173, June, 1975.

[12] J. B. Goodenough, Ada Compiler Validation: An Example of Software Testing Theory and Practice, Wang Institute of Graduate Studies Technical Report TR-86-07, July, 1986.

[13] M. Gordon, The Denotational Description of Programming Languages. New York: Springer-Verlag, 1979.

[14] B. Hetzel, The Complete Guide to Software Testing, 2nd ed. Wellesley: QED Information Sciences, 1988.

[15] R. House, "Comments on Program Specification and Testing," Comm. ACM, vol. 23, no. 6, pp. 324-331, June, 1980. 
[16] W.E. Howden, "A Survey of Dynamic Analysis Methods," in Tutorial: Software Testing \& Validation Techniques, 2nd ed., pp. 209-231, E. Miller and W.E. Howden, Eds. Washington, DC: IEEE Computer Society Press, 1981.

[17] R.E. Kalman, P.L. Falb, and M.A. Arbib, Topics in Mathematical System Theory. New York, McGraw-Hill:1969.

[18] L. Lamport, On the Proof of Correctness of a Calendar Program, Comm. ACM, vol. 22, no. 10, pp. 554-556, October 1979,

[19] E. J. McCluskey, Logic Design Principles. New York:Prentice-Hall, 1986.

[20] G.J. Meyers, The Art of Software Testing. New York: Wiley, 1979.

[21] G.J. Meyers, "A Controlled Experiment in Program Testing and Code Walkthroughs/Inspections," Comm. ACM, vol. 21, no. 9, pp. 760-768, September, 1978.

[22] P. Naur, "Programming by Action Clusters," BIT, vol. 9, no. 3, pp. 250-258, 1969.

[23] S. Reddy, "Easily Testable Realizations for Logic Functions," IEEE Trans. Comput., vol. C21, pp. 1183-1188, November, 1972.

[24] D. Scott and C. Strachey, "Towards a Mathematical Semantics for Programming Languages," Proc. of the Symposium on Computers and Automata, J. Fox, Ed., New York, Polytechnic Institute of Brooklyn Press: 1971.

[25] M.L. Shooman, Software Engineering: Design, Reliability, and Management. New York: McGraw-Hill, 1983.

[26] J. Stoy, Denotational Semantics: The Scott-Strachey Approach to Programming Language Theory. Cambridge: MIT Press, 1977.

[27] L. Tsalalikhin, "Dialog with a Tester (Architecture and Functions of One Unit Test Facility)," Proceedings of the 1986 Workshop on Software Testing, pp. 51-60, IEEE Computer Society.

[28] M. Watkins, "A Technique for Testing Command and Control Software," Comm. ACM, vol. 25, no. 4, pp. 228-232, April, 1982.

[29] E.J. Weyuker and S. Rapps, "Selecting Software Tests Using Data Flow Information," IEEE Trans. Software Eng., vol. SE-11, pp. 367-375, April, 1985.

[30] E.J. Weyuker, "Axiomatizing Software Test Data Adequacy," IEEE Trans. Software Eng., vol. SE-12, pp. 1128-1138, December, 1986. 
Testability of Software Components

[31] S.H Zweben and J.S. Gourlay, "On the Adequacy of Weyuker's Test Data Adequacy Axioms," IEEE Trans. Software Eng., vol. SE-15, pp. 496-501, April, 1989.

[32] ANSI/IEEE Std 829-1983 (Standard for Software Test Documentation) and ANSI/IEEE Std 1008-1987 (Standard for Software Unit Testing), in Software Engineering Standards, SH12534, 3rd ed., New York, IEEE: 1989. 


\section{APPENDIX}

Denotational semantics provides a rigorous mathematical description of the domains and function spaces in applicative, conventional (von Neumann), and functional programming languages [1]. For our purposes, we explicitly specify the input, output and state transformations for our model language to define a direct semantics. The semantics of most conventional programming languages are usually specified in terms of a continuation semantics. Continuations are used to represent the meanings of constructs that depend on the rest of the program: state transformations are specified indirectly [13]. In our discussion of testability, it is easier to consider direct semantics.

\section{A. Specification of Test Execution Semantics}

A direct semantics requires the specification of syntactic domains, syntactic clauses, semantic domains, semantic functions, and semantic clauses. Domains are sets that support recursive definition [24].

Syntactic domains and clauses specify the form of our language. We assume that the following syntactic domains are defined:

Ide

The domain of programming language identifiers includes I, P, F, O,...

Type

The domain of types supported by the programming language includes INTEGER, FLOAT, ...

Exp

The domain of expressions.

\section{Com}

The domain of commands.

\section{Def}

The domain of definitions.

In the sequel, we denote

$\mathrm{D} \in$ Def

$\mathrm{T} 1, \mathrm{~T} 2, \ldots, \mathrm{Tn}, \ldots \mathrm{T}^{*}, \ldots \in$ Type

F, P, I1, I2, ... In, .. O1, O2, ... On,... $\in$ Ide 


\section{Testability of Software Components}

Here, $\mathrm{a} \in \mathrm{S}$ denotes that $\mathrm{a}$ is an element of a set $\mathrm{S}$. Syntactic clauses are used to specify the form of these domains. We specify the forms of $n$-ary expression procedures and command procedures in a variant of BNF (we follow the notation specified in $[13,26]$ ).

Expression procedure abstracts define "functions" for $\mathrm{n}$ arguments, $\mathrm{n}=0$ :

$$
\mathrm{D}::=\text { function } \mathrm{F}\left(\mathrm{I} 1: \text { in } \mathrm{T} 1 ; \mathrm{I} 2: \text { in } \mathrm{T} 2 ; \ldots, \mathrm{In}: \text { in } \mathrm{Tn} ; \text { ) return } \mathrm{T}^{*}\right. \text {; }
$$

Similarly, command procedure abstracts define command procedures for $\mathrm{n}$ input arguments and $\mathrm{m}$ output arguments, $\mathrm{n}, \mathrm{m}=0$ :

$$
\mathrm{D}::=\text { procedure } \mathrm{P}\left(\mathrm{I} 1: \text { in } \mathrm{T} 1 ; \ldots, \mathrm{In}: \text { in } \mathrm{Tn} ; \mathrm{O} 1: \text { out } \mathrm{T}^{*} ; \ldots \mathrm{Om} \text { : out } \mathrm{Tm}^{*}\right)
$$

The syntax of expressions and commands includes the evaluation of expression procedures and the execution of command procedures:

For

$$
\begin{aligned}
& \text { E, E1, E2, .., En, .. Exp } \\
& \text { E ::=F }()|\mathrm{F}(\mathrm{E} 1)| \mathrm{F}(\mathrm{E} 1, \mathrm{E} 2)|\ldots| \mathrm{F}(\mathrm{E} 1, \mathrm{E} 2, \ldots, \mathrm{En}) \mid \ldots \\
& \mathrm{C}::=\mathrm{P}() ;|\mathrm{P}(\mathrm{E} 1) ;| \mathrm{P}(\mathrm{O} 1) ;|\mathrm{P}(\mathrm{E} 1, \mathrm{O} 1) ;| \ldots|\mathrm{P}(\mathrm{E} 1, \ldots, \mathrm{En}, \mathrm{O} 1, \ldots, \mathrm{Om}) ;| \ldots
\end{aligned}
$$

Semantic domains and clauses specify the denotations of our language. We assume that the following semantic domains are defined:

Err

A domain of error values.

Val

This denotes the domain of values. We assume that the domain of values includes the domain Err of error values.

Env $=$ Ide $\rightarrow$ Val

The environment is the domain of all functions from the domain of identifiers to the domain of values. To simplify the discussion, we assume all identifiers are bound to a value (which may be an error value). In our notation, A? B denotes the set of functions from A to B.

\section{TypeEnv $=$ Ide $\rightarrow$ Type}

The environment of types is the domain of all functions from the domain of identifiers to the domain of types. 


\section{State $=[$ Env + TypeEnv $] \times$ Input $\times$ Output + Err}

The states are denoted by the domain of all 3 -tuples $(\mathrm{u}, \mathrm{i}, \mathrm{o})$, where $\mathrm{u} \in \mathbf{E n v}+$ TypeEnv, $\mathrm{i} \in \mathbf{I n p u t}$ is an input domain and $\mathrm{o} \in$ Output is an output domain. The state also includes the domain Err which is used to capture program errors. State transformations are caused by the execution of commands and the evaluation of expressions. In our notation, A x B denotes a set of pairs (the cartesian product of sets A and B), and A + B denotes set union.

Semantic functions and clauses provide the semantics of our language by specifying the correspondence between the syntax and literal values (the denotations). We assume that the following semantic functions are defined:

The semantics of expression evaluation is specified by

$$
\mathbf{E}: \operatorname{Exp} \rightarrow(\text { State } \rightarrow(\text { Val X State }))
$$

This means that the evaluation of an expression depends on the state, and results in a value and a new state. For example, the evaluation of the expression procedure $F(E)$ is denoted by

$$
\mathbf{E}[F(E)] s=\left(v, s^{\prime}\right) \text {, for } v \in \text { Val and } s, s^{\prime} \in \text { State }
$$

To reduce the number of parentheses, it is convenient to place brackets ( [ ] ) around elements of a syntactic domain.

In most programming languages, the result of evaluating an expression procedure affects the state, as in

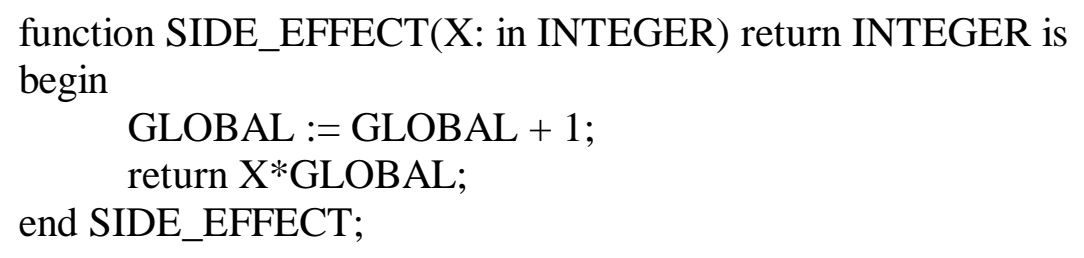

For convenience, we define a projection function getval

$$
\text { getval: Val x State } \rightarrow \text { Val }
$$

Command execution depends on the state, and results in a new state:

$$
\text { C: Com } \rightarrow \text { State } \rightarrow \text { State }
$$

For example, the evaluation of the command procedure $\mathrm{P}(\mathrm{E}, \mathrm{O})$ is denoted by 


$$
\text { C }\left[\mathrm{P}(\mathrm{E}, \mathrm{O}) ; \mathrm{s}=\mathrm{s}^{\prime} \text {, for } \mathrm{s}, \mathrm{s}^{\prime} \in\right. \text { State }
$$

Context evaluation for expressions depends on the state (the TypeEnv) and results in the type of the expression:

$$
\text { T: } \operatorname{Exp} \rightarrow \text { State } \rightarrow \text { Type }
$$

For example, the context evaluation of the expression procedure $\mathrm{F}(\mathrm{E})$ is denoted by

$$
\mathbf{T}[F(E)] s=t \text {, where } s \in \text { State and } t \in \text { Type }
$$

\section{B. Observability}

Given an expression procedure defined by definition

$$
\text { D::= function F(I: in T) return } T^{*} \text {; }
$$

$\mathrm{F}$ is observable if for any $\mathrm{s}_{1}$ and $\mathrm{s}_{2}$, distinct outputs are generated from distinct inputs:

$$
\begin{aligned}
& \operatorname{getval}\left(\mathbf{E}\left[F\left(E_{1}\right)\right] s_{1}\right)=\operatorname{getval}\left(\mathbf{E}\left[F\left(E_{2}\right)\right] s_{2}\right. \\
& \text { if } \\
& \operatorname{getval}\left(\mathbf{E}\left[E_{1}\right] s_{1}\right)=\operatorname{getval}\left(\mathbf{E}\left[E_{2}\right] s_{2}\right)
\end{aligned}
$$

We note that in general, the evaluation of the output of $\mathrm{F}$ is a function $\mathbf{F} *$ of the evaluation of the input and the states, such that for $\mathbf{E}[\mathrm{E}] \mathrm{s}=\left(\mathrm{i}, \mathrm{s}^{*}\right)$ and $\mathbf{E}[\mathrm{F}(\mathrm{E})] \mathrm{s}=\left(\mathrm{o}, \mathrm{s}^{+}\right)$

$$
\mathrm{o}=\mathbf{F} *\left(\mathrm{i}, \mathrm{s}^{*}\right)
$$

In other words, the evaluation of the function procedure may have a side effect in changing the state.

Observability implies that the output value of $\mathrm{F}$ is a function $\mathbf{F}$ of the input expression:

or

$$
\operatorname{getval}(\mathbf{E}[F(E)] s)=\mathbf{F}(\operatorname{getval}(\mathbf{E}[E] \mathrm{s}))
$$

$$
\mathrm{o}=\mathbf{F} \text { (i) }
$$


For example, the following expression procedure is not observable:

function $\mathrm{F}(\mathrm{X}$ : in INTEGER) return INTEGER is

begin

return $\mathrm{X}^{*}$ GLOBAL_VARIABLE;

end F;

Subsequent calls to the function $\mathrm{F}$ with the same arguments can yield different results. In an environment where GLOBAL_VARIABLE $=0, \mathrm{~F}(3)=0$. If the environment is changed after this function call where GLOBAL_VARIABLE $=2$, then $F(3)=6$. We note that

$$
\begin{aligned}
\operatorname{getval}(\mathbf{E}[\mathrm{F}(\mathrm{E})] \mathrm{s}) & \\
& =\operatorname{getval}(\mathbf{E}[\operatorname{GLOBAL}] \mathrm{s}) * \operatorname{getval}(\mathbf{E}[\mathrm{E}] \mathrm{s}) \\
& =\mathbf{F}^{*}(\mathrm{~g}, \mathrm{e}) \neq \mathbf{F}(\mathrm{e})
\end{aligned}
$$

Observability can be defined for an expression procedure

$$
\mathrm{D}::=\text { function } \mathrm{F}\left(\mathrm{I} 1: \text { in } \mathrm{T} 1 ; \mathrm{I} 2 \text { : in } \mathrm{T} 2 ; \ldots, \mathrm{In}: \text { in } \mathrm{Tn} ; \text { ) return } \mathrm{T}^{*}\right. \text {; }
$$

$\mathrm{F}$ is observable if distinct outputs are generated from distinct inputs:

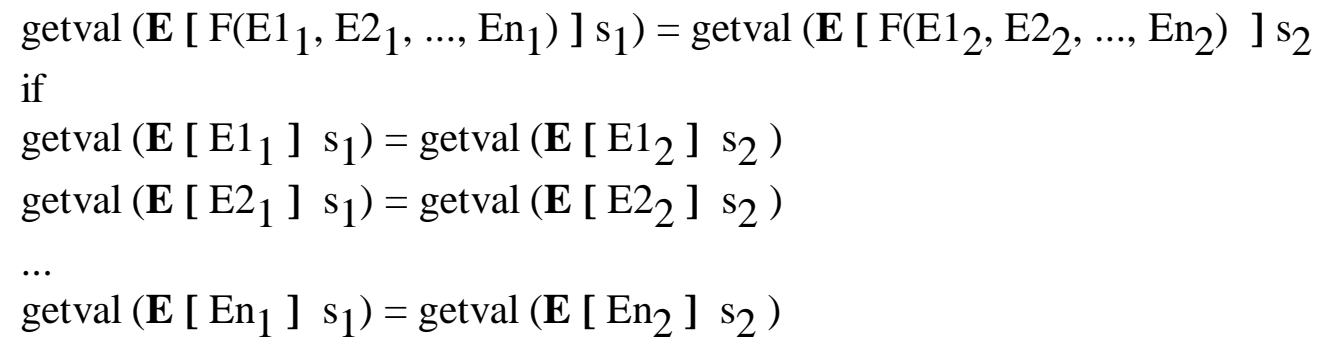

Similar definitions of observability are defined for commands. The execution of a Command Procedure is observable if distinct outputs are generated from distinct inputs. For explicit outputs bound in the procedure abstract, the execution of $\mathrm{C}(\mathrm{E}, \mathrm{O})$ is output observable where for $\mathrm{C}\left(\mathrm{E}_{1}\right.$, $\left.\mathrm{O}_{1}\right)$ and $\mathrm{C}\left(\mathrm{E}_{2}, \mathrm{O}_{2}\right)$

$\mathbf{E}\left[\mathrm{O}_{1}\right] \mathrm{s}_{1}=\mathbf{E}\left[\mathrm{O}_{2}\right] \mathrm{s}_{2}$ if $\operatorname{getval}\left(\mathbf{E}\left[\mathrm{E}_{1}\right] \mathrm{s}_{1}\right)=\operatorname{getval}\left(\mathbf{E}\left[\mathrm{E}_{2}\right] \mathrm{s}_{2}\right)$ 


\section{Testability of Software Components}

\section{Controllability}

Given a state s, the domain of values of the evaluation map for expressions is usually a subset of the target type:

$\mathrm{T}[\mathrm{F}] \mathrm{s} \supseteq\{\operatorname{getval}(\mathbf{E}[\mathrm{F}(\mathrm{E})] \mathrm{s}) \mid$ for all $\mathrm{E} \in \mathbf{E x p}\}$

For example, given the expression procedure $\mathrm{G}$

function $\mathrm{G}(\mathrm{X}$ : in POSITIVE) return POSITIVE is

begin

return $\mathrm{X} \bmod 3$;

end;

$\mathrm{G}$ is observable since $\mathrm{G}$ returns a subset of the type POSITIVE, for every state s:

POSITIVE $\supseteq\{\operatorname{getval}(\mathbf{E}[\mathrm{G}(\mathrm{E})] \mathrm{s})$, for all $\mathrm{E} \in \mathbf{E x p}\}=\{0,1,2\}$

An evaluation of expression procedure $\mathrm{F}(\mathrm{E})$ is controllable if, for any state $\mathrm{s}$, the domain of values of the evaluation map equals its type:

$$
\mathrm{T}[\mathrm{F}] \mathrm{s}=\in\{\operatorname{getval}(\mathbf{E}[\mathrm{F}(\mathrm{E})] \mathrm{s}) \mid \text { for all } \mathrm{E} \in \operatorname{Exp}\}
$$

For example, the expression procedure $\mathrm{G}$ is not controllable.

\section{Observable Extensions}

Let expression procedure $\mathrm{F}$ have definition abstract

$$
\mathrm{D}::=\text { function } \mathrm{F}(\mathrm{E}: \text { in } \mathrm{T}) \text { return } \mathrm{T}^{*} \text {; }
$$

F has an observable extension if there exists an observable expression procedure Fo, with definition abstract

$$
\mathrm{D}::=\text { function } \mathrm{Fo}\left(\mathrm{E}_{1}: \text { in } \mathrm{T}_{1} ; \mathrm{E}_{2}: \text { in } \mathrm{T}_{2} ; \ldots ; \mathrm{E}_{\mathrm{n}}: \text { in } \mathrm{T}_{\mathrm{n}}\right) \text { return } \mathrm{T}^{*} \text {; }
$$

such that for all $\mathrm{E} \in \mathbf{E x p}$ and any state s, there exists a state s* such that

$$
\operatorname{getval}(\mathbf{E}[\mathrm{F}(\mathrm{E})] \mathrm{s})=\operatorname{getval}\left(\mathbf{E}\left[\mathrm{Fo}\left(\mathrm{E}_{1}, \mathrm{E}_{2}, \ldots, \mathrm{E}_{\mathrm{n}}\right)\right] \mathrm{s}^{*}\right)
$$


For example, the following expression procedure Fo is an observable extension of F:

function Fo (X: in INTEGER; G: in INTEGER) return INTEGER is begin return $X^{*} \mathrm{G}$ end Fo;

Fo is observable, since

$$
\begin{aligned}
\operatorname{getval}(\mathbf{E}[\mathrm{Fo} & \left.\left.\left(\mathrm{A}_{1}, \mathrm{~A}_{2}\right)\right] \mathrm{s}\right) \\
& =\operatorname{getval}\left(\mathbf{E}\left[\mathrm{A}_{1}\right] \mathrm{s}\right)^{*} \operatorname{getval}\left(\mathbf{E}\left[\mathrm{A}_{2}\right] \mathrm{s}\right) \\
& =\operatorname{getval}\left(\mathbf{E}\left[\mathrm{Fo}_{(}\left(\mathrm{B}_{1}, \mathrm{~B}_{2}\right)\right] \mathrm{s}\right) \\
& =\operatorname{getval}\left(\mathbf{E}\left[\mathrm{B}_{1}\right] \mathrm{s}\right)^{*} \operatorname{getval}\left(\mathbf{E}\left[\mathrm{B}_{2}\right] \mathrm{s}\right)
\end{aligned}
$$

as long as both

$$
\begin{aligned}
& \operatorname{getval}\left(\mathbf{E}\left[A_{1}\right] \mathrm{s}\right)=\operatorname{getval}\left(\mathbf{E}\left[B_{1}\right] \mathrm{s}\right) \\
& \operatorname{getval}\left(\mathbf{E}\left[A_{2}\right] \mathrm{s}\right)=\operatorname{getval}\left(\mathbf{E}\left[B_{2}\right] \mathrm{s}\right)
\end{aligned}
$$

Moreover, for all states we can set

$$
\operatorname{getval}\left(\mathbf{E}[\text { GLOBAL] })=\operatorname{getval}\left(\mathbf{E}\left[E_{2}\right] \mathrm{s}\right)\right.
$$

so that

$$
\begin{aligned}
\operatorname{getval}(\mathbf{E}[\mathrm{F}(\mathrm{E})] \mathrm{s}) & \\
& =\operatorname{getval}(\mathbf{E}[\mathrm{GLOBAL}] \mathrm{s}) * \operatorname{getval}(\mathbf{E}[\mathrm{E}] \mathrm{s}) \\
& =\operatorname{getval}\left(\mathbf{E}\left[\mathrm{E}_{1}\right] \mathrm{s}\right) * \operatorname{getval}\left(\mathbf{E}\left[\mathrm{E}_{2}\right] \mathrm{s}\right) \\
& =\operatorname{getval}\left(\mathbf{E}\left[\mathrm{Fo}\left(\mathrm{E}_{1}, \mathrm{E}_{2}\right)\right] \mathrm{s}\right)
\end{aligned}
$$

Let command procedure $\mathrm{P}$ have definition abstract

$$
\mathrm{D}::=\text { procedure } \mathrm{P}\left(\mathrm{E}: \text { in } \mathrm{T} \text {; O: out } \mathrm{T}^{*}\right) \text {; }
$$

$\mathrm{P}$ has an observable extension of $\mathrm{P}$ if there exists an observable command procedure $\mathrm{P}^{\mathrm{O}}$, with definition abstract

$$
\mathrm{D}::=\text { procedure } \mathrm{P}^{\mathrm{O}}\left(\mathrm{E}_{1}: \text { in } \mathrm{T}_{1} ; \mathrm{E}_{2}: \text { in } \mathrm{T}_{2} ; \ldots ; \mathrm{E}_{\mathrm{n}} \text { : in } \mathrm{T}_{\mathrm{n}} ; \mathrm{O}^{*} \text { : out } \mathrm{T}^{*}\right) \text {; }
$$


such that for all $\mathrm{E} \in \mathbf{E x p}$ and any state s, there exists a state s* such that

$$
\begin{aligned}
& \mathbf{E}\left[\mathrm{O}^{*}\right] \mathrm{r}^{*}=\mathbf{E}[\mathrm{O}] \mathrm{r} \\
& \text { where } \\
& \qquad \begin{aligned}
\mathrm{r} * & =\mathbf{C}\left[\mathrm{P}^{\mathrm{O}}\left(\mathrm{E}_{1}, \mathrm{E}_{2}, \ldots, \mathrm{E}_{\mathrm{n}}, \mathrm{O}^{*}\right) ;\right] \mathrm{s}^{*} \\
\mathrm{r} & =\mathbf{C}[\mathrm{P}(\mathrm{E}, \mathrm{O}) ;] \mathrm{s}
\end{aligned}
\end{aligned}
$$

\section{Controllable Extensions}

F has a controllable extension if there exists a controllable expression procedure $\mathrm{F}^{\mathrm{C}}$, with definition abstract

$$
\mathrm{D}::=\text { function } \mathrm{F}^{\mathrm{c}}\left(\mathrm{E}_{1}: \text { in } \mathrm{T}_{1} ; \mathrm{E}_{2}: \text { in } \mathrm{T}_{2} ; \ldots ; \mathrm{E}_{\mathrm{n}}: \text { in } \mathrm{T}_{\mathrm{n}}\right) \text { return } \mathrm{T}^{\mathrm{c}}
$$

such that for all $\mathrm{E} \in \mathbf{E x p}$ and any state $\mathrm{s}$, there exists a state $\mathrm{s}^{*}$ such that

$$
\operatorname{getval}(\mathbf{E}[\mathrm{F}(\mathrm{E})] \mathrm{s})=\operatorname{getval}\left(\mathbf{E}\left[\mathrm{F}^{\mathrm{c}}\left(\mathrm{E}_{1}, \mathrm{E}_{2}, \ldots, \mathrm{E}_{\mathrm{n}}\right)\right] \mathrm{s}^{*}\right)
$$

For example, the following expression procedure $\mathrm{G}^{\mathrm{c}}$ is a controllable extension of $\mathrm{G}$ (defined in Section II.C):

type Small is POSITIVE range 0..2;

function $\mathrm{G}^{\mathrm{C}}$ (X: in POSITIVE) return SMALL is

begin

return SMALL'(X mod 3);

end $\mathrm{G}^{\mathrm{c}}$;

$\mathrm{F}^{\mathrm{C}}$ is controllable, since

$$
\left\{\operatorname{getval}\left(\mathbf{E}\left[\mathrm{F}^{\mathrm{c}}(\mathrm{E})\right] \mathrm{s}\right) \mid \text { for all } \mathrm{E} \in \operatorname{Exp}\right\}=\{0,1,2\}=\mathrm{SMALL}=\mathrm{T}\left[\mathrm{F}^{\mathrm{c}}\right] \mathrm{s}
$$

Moreover, for all states s,

$$
\mathbf{E}[\mathrm{G}(\mathrm{E})] \mathrm{s}=\mathbf{E}\left[\mathrm{G}^{\mathrm{c}}(\mathrm{E})\right] \mathrm{s}
$$


Testability of Software Components

$\mathrm{P}$ has a controllable extension if there exists a controllable command procedure $\mathrm{P}^{\mathrm{C}}$, with definition abstract

$$
\mathrm{D}::=\text { procedure } \mathrm{P}^{\mathrm{c}}\left(\mathrm{E}_{1}: \text { in } \mathrm{T}_{1} ; \mathrm{E}_{2}: \text { in } \mathrm{T}_{2} ; \ldots ; \mathrm{E}_{\mathrm{n}}: \text { in } \mathrm{T}_{\mathrm{n}} ; \mathrm{O}^{*} \text { : out } \mathrm{T}^{+}\right) \text {; }
$$

such that for all E $\in$ Exp and any state s, there exists a state s* such that

$$
\mathbf{E}\left[\mathrm{O}^{*}\right] \mathrm{r}^{*}=\mathbf{E}[\mathrm{O}] \mathrm{r}
$$

where

$$
\begin{aligned}
& r^{*}=\mathbf{C}\left[\mathrm{P}^{\mathrm{c}}\left(\mathrm{E}_{1}, \mathrm{E}_{2}, \ldots, \mathrm{E}_{\mathrm{n}}, \mathrm{O}^{*}\right) ;\right] \mathrm{s}^{*} \\
& \mathrm{r}=\mathbf{C}[\mathrm{P}(\mathrm{E}, \mathrm{O}) ;] \mathrm{s}
\end{aligned}
$$

